\title{
Monomial Gorenstein algebras and the stably Calabi-Yau property
}

\section{Ana Garcia Elsener ${ }^{1}$ (D)}

Received: 7 August 2018 / Accepted: 2 July 2020 / Published online: 14 August 2020

(C) The Author(s) 2020

\begin{abstract}
A celebrated result by Keller-Reiten says that 2-Calabi-Yau tilted algebras are Gorenstein and stably 3-Calabi-Yau. This note shows that the converse holds in the monomial case: a 1-Gorenstein monomial algebra and stably 3-Calabi-Yau is 2-Calabi-Yau tilted, moreover is Jacobian. We study the case of other stably Calabi-Yau Gorenstein monomial algebras.
\end{abstract}

Keywords 2-Calabi-Yau tilted algebras · Monomial · Jacobian algebras ·

Gorenstein-projective $\cdot$ Stably Calabi-Yau

Mathematics Subject Classification (2010) $16 \mathrm{G} 20 \cdot 16 \mathrm{G} 50$

\section{Introduction}

Cluster-tilted algebras are inspired by Fomin-Zelevinsky cluster algebras, a class of commutative algebras that was introduced in [15], also they are related to tilted algebras [19]. Whereas tilted algebras are defined as endomorphism algebras of tilting objects over a hereditary algebra, a cluster-tilted algebra is the endomorphism algebra of a cluster-tilting object in a triangulated category called the cluster category of a hereditary algebra. The crucial step to define a cluster algebra, and the way to obtain new cluster-tilted algebras from old is called mutation. The mutation algorithm takes a quiver (or matrix) and transforms it into a new one, and the cluster-tilting mutation process takes a certain finite dimensional algebra and defines a new one that is closely related to the original. All the algebras given by this operation share interesting homological properties and their module categories are very close to each other. Cluster categories and cluster-tilted algebras were introduced in $[9,10,12]$.

Communicated by:Presented by: Steffen Koenig

Ana Garcia Elsener

ana.garcia-elsener@uni-graz.at

1 Institut für Mathematik und Wissenschaftliches Rechnen, Universität Graz, Heinrichstraße 36, A-8010 Graz, Austria 
The clear interaction between cluster algebras and cluster tilted algebras has motivated hundreds of works. Some of them are devoted to study other 'cluster like' categories and their cluster-tilting objects. So is the case of 2-Calabi-Yau tilted algebras which are obtained by replacing the cluster category by a 2-Calabi-Yau triangulated category. The definition of 2-Calabi-Yau tilted algebra appeared explicitly in [8]. The reader can also find important references in [27]. One of the main articles on the theory is the one by Keller-Reiten [21] where it is proved that 2-Calabi-Yau tilted algebras are 1-Iwanaga-Gorenstein and stably 3-Calabi-Yau, i. e. with a 3-Calabi-Yau stable category of Gorenstein projective objects (singularity category).

A large family of 2-Calabi-Yau tilted algebras are Jacobian algebras of a quiver with potential $(Q, W)$. Jacobian algebras are defined by applying cyclic derivatives to a quiver with potential (a generalization called quiver with hyperpotential can be considered [23]). As before, new quivers with potential can be obtained from old ones by mutation, see [14]. The 2-Calabi-Yau category that allows us to obtain Jacobian algebras as endomorphism algebras of cluster-tilting objects is called the generalized cluster category $\mathcal{C}_{(Q, W)}$ and was introduced by Amiot [1] building on previous works by Keller and Ginzburg.

On the other hand, in recent years several authors studied Georenstein monomial algebras and their singularity categories [13, 25]. These articles show that combinatorics behind Gorenstein projective stable categories is nice when we are dealing with 1-Iwanaga-Gorenstein monomial algebras.

As mentioned above, we know that Jacobian algebras are 2-Calabi-Yau tilted, and also that 2-Calabi-Yau tilted algebras are 1-Iwanaga-Gorenstein and stably 3-Calabi-Yau. But are these conditions equivalent?

In the case of monomial algebras, this article uses the combinatorics of Gorenstein projective stable categories to show that the answer is affirmative.

The main result of the article is the following:

Theorem 1 (Theorem 3.5) Let $k$ be an algebraically closed field of characteristic zero and $\Lambda=k Q /$ I a monomial $k$-algebra. The following are equivalent:

(1) $\Lambda$ is 2-Calabi-Yau tilted,

(2) $\Lambda$ is a 1-Iwanaga-Gorenstein algebra and stably 3-Calabi-Yau,

(3) $\Lambda$ is Jacobian.

With the consideration of hyperpotentials instead of potentials, the condition chark $=0$ can be removed.

Jacobian algebras include a well known class of gentle 2-Calabi-Yau tilted algebras whose quivers with potential can be obtained from triangulations of unpunctured surfaces, see [3]. This family includes the cluster-tilted algebras of type $\mathbb{A}$ and $\tilde{\mathbb{A}}$. A related family of gentle algebras, that also have a geometric realization via unpunctured surfaces, are the $m$-cluster-tilted algebras of types $\mathbb{A}$ and $\tilde{\mathbb{A}}$, see $[5,18,28]$. This family is a subfamily of gentle algebras defined by a bound quiver that can be realized as a dual quiver obtained from $(m+2)$-angulations as well. In Section 4 we consider a family of algebras arising from surfaces that generalize the class of $m$-cluster tilted algebras of types $\mathbb{A}$ and $\tilde{\mathbb{A}}$. The main result of this section characterizes their Gorenstein projective modules.

Theorem 2 (Theorem 4.5) Let $\Lambda_{\mathcal{T}}=k Q_{\mathcal{T}} / I_{\mathcal{T}}$ be an algebra arising from a $(m+$ 2)-angulation and $N$ a $\Lambda_{\mathcal{T}}$-module. Then $N$ is Gorenstein projective if and only if 
$\Omega^{m+1} \tau N \simeq N$ over $\bmod \Lambda_{\mathcal{T}}$. In particular, this holds for $m$-cluster tilted algebras of type $\mathbb{A}$ and $\tilde{\mathbb{A}}$.

\section{Preliminaries}

Throughout these notes, we consider $k$-algebras where $k$ is an algebraically closed field of characteristic zero (if the contrary is not stated). Let $Q=\left(Q_{0}, Q_{1}\right)$ be a finite quiver, where $Q_{0}$ is the set of vertices and $Q_{1}$ the set of arrows. Let $s, t: Q_{1} \rightarrow Q_{0}$ be the functions that indicate the source and the target of each arrow, respectively. We will only consider finite dimensional basic $k$-algebras. Every finite dimensional basic $k$-algebra $\Lambda$ is isomorphic to a quotient $k Q / I$, where $I$ is an admissible ideal. The pair $(Q, I)$ is called a bound quiver [4, Chapter III].

Let $\Omega$ be the usual syzygy operator, $\tau$ the Auslander-Reiten (AR) translation, and $D=$ $\operatorname{Hom}_{k}(-, k)$. Denote by $\mathcal{D}^{b}\left(\mathbb{A}_{m}\right)$ the derived category of a hereditary algebra of Dynkin type $\mathbb{A}_{m}$.

Definition 2.1 A $k$-algebra $\Lambda$ is Iwanaga-Gorenstein if inj.dim $\Lambda=\operatorname{proj.dim} D\left(\Lambda^{o p}\right) \leq d$ for some non-negative integer $d$. In this case we say that $\Lambda$ is $d$-Iwanaga-Gorenstein.

Remark 2.2 For a $d$-Iwanaga-Gorenstein algebra $\Lambda$, a $\Lambda$-module $M$ is Gorensteinprojective if and only if $M$ is a $d$-th syzygy, see [6, Proposition 6.20]. In this case each $\Lambda$-module either has infinite projective dimension or has projective dimension at most $d$. The stable subcategory of Gorenstein-projetive modules, also called singularity category, $\underline{\mathrm{GP}}(\Lambda)$ is triangulated, with shift given by the formal inverse of $\Omega$.

An algebra $\Lambda=k Q / I$ is monomial if all the generators of $I$ are paths $\alpha_{1} \ldots \alpha_{t}$. Denote by $F$ the minimal set of paths generating $I$. Following [25, Section 6.2], when $k Q / I$ is monomial and 1-Iwanaga-Gorenstein the elements of $F$ are very particular: $F$ is a disjoint union $\coprod_{i=1}^{m} F_{i}$ where the subset $F_{i}$ consists of all sub-paths over a cyclic path $c_{i}=x_{1} \stackrel{\alpha_{1}}{\longrightarrow}$ $\cdots \rightarrow x_{n_{i}} \stackrel{\alpha_{n_{i}}}{\longrightarrow} x_{1}$ of certain common length. The cycles $c_{i}$ are considered up to cyclic equivalence, and without arrow repetition. Denote by $\mathcal{C}(\Lambda)$ the set of cycles $c_{1}, \ldots, c_{m}$ that contain the paths in $F_{1}, \ldots, F_{m}$ respectively, by $n_{i}$ the length of the cycle $c_{i}$ and by $r_{i}$ the (common) length of paths in $F_{i}$. We refer to the generators in $F$ as zero-relations. By [25, Lemma 6.12], two different cycles $c_{i}, c_{j}$ in $\mathcal{C}(\Lambda)$ do not share arrows.

Example 2.3 Let $Q$ be the quiver with ideal $I=<\alpha \beta \gamma, \beta \gamma \delta, \gamma \delta \alpha, \delta \alpha \beta, \lambda^{4}>$

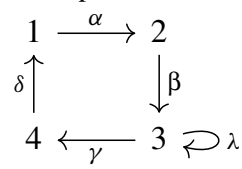

Then $F=F_{1} \bigsqcup F_{2}$ with $F_{1}=\{\alpha \beta \gamma, \beta \gamma \delta, \gamma \delta \alpha, \delta \alpha \beta\}, F_{2}=\left\{\lambda^{4}\right\}$. We have $c_{1}=\alpha \beta \gamma \delta$ and $c_{2}=\lambda$, so $n_{1}=4, n_{2}=1, r_{1}=3$ and $r_{2}=4$.

Lemma 2.4 [25, Theorem 6.16] Let $\Lambda=k Q / I$ be a 1-Iwanaga-Gorenstein monomial algebra. The singularity category $\mathrm{GP}(\Lambda)$ is equivalent to the coproduct $\bigsqcup_{c_{i} \in \mathcal{C}(\Lambda)} \mathcal{D}^{b}\left(\mathbb{A}_{r_{i}-1}\right) / \tau^{n_{i}}$. 


\subsection{Gentle algebras}

We recall the definition of gentle algebra. By [17] it is known that gentle algebras are Iwanaga-Gorenstein.

Definition 2.5 A $k$-algebra $\Lambda=k Q / I$ is gentle if

(g1) For each $x_{0} \in Q_{0}$ there are at most two arrows with source $x_{0}$, and at most two arrows with target $x_{0}$,

(g2) the ideal $I$ is generated by paths of length 2 ,

(g3) for each $\beta \in Q_{1}$ there is at most one arrow $\alpha \in Q_{1}$ and at most one arrow $\gamma$ such that $\alpha \beta \in I$ and $\beta \gamma \in I$, and

(g4) for each $\beta \in Q_{1}$ there is at most one arrow $\alpha$ and at most one arrow $\gamma$ such that $\alpha \beta \notin I$ and $\beta \gamma \notin I$.

An algebra $\Lambda=k Q / I$, where $I$ is generated by paths and $(Q, I)$ satisfies the two conditions (g1) and (g4), is called a string algebra. Thus every gentle algebra is a string algebra. A string in $\Lambda$ is by definition a reduced walk $w$ in $Q$ avoiding the zero-relations, thus $w$ is a sequence $x_{1} \stackrel{\alpha_{1}}{\longleftrightarrow} x_{2} \stackrel{\alpha_{2}}{\longleftrightarrow} \cdots \stackrel{\alpha_{n}}{\longleftrightarrow} x_{n+1}$ where the $x_{i}$ are vertices of $Q$ and each $\alpha_{i}$ is an arrow between the vertices $x_{i}$ and $x_{i+1}$ in either direction such that there is no $\stackrel{\beta}{\longrightarrow} \stackrel{\beta}{\longleftarrow}$, no $\stackrel{\beta}{\longleftarrow} \stackrel{\beta}{\longrightarrow}$, and no $\stackrel{\beta_{1}}{\longleftarrow} \ldots \stackrel{\beta_{t}}{\longleftarrow}$ or $\stackrel{\beta_{1}}{\longrightarrow} \ldots \stackrel{\beta_{t}}{\longrightarrow}$ with $\beta_{1} \ldots \beta_{t} \in I$. If the first and the last vertex of $w$ coincide, then the string is cyclic. A band is a cyclic string $b$ such that each power $b^{n}$ is a cyclic string but $b$ is not a power of some string. The classification of indecomposable modules over a string algebra $\Lambda=k Q / I$ is given by Butler-Ringel [11] in terms of strings and bands in $(Q, I)$. Each string $w$ defines an indecomposable module $M(w)$, called a string module, and each band $b$ defines a family of indecomposable modules $M(b, \lambda, n)$, called band modules, with parameters $\lambda \in k$ and $n \in \mathbb{N}$.

Definition 2.6 Let $\Lambda=k Q / I$ be a gentle algebra.

(a) A cycle $x_{1} \stackrel{\alpha_{1}}{\rightarrow} \cdots \rightarrow x_{n} \stackrel{\alpha_{n}}{\rightarrow} x_{1}$ is saturated if $\alpha_{i} \alpha_{i+1} \in I$, for $i \in \mathbb{Z} / n \mathbb{Z}$. In particular, a saturated loop is an arrow $\delta$ such that $s(\delta)=t(\delta)$ and $\delta^{2} \in I$.

(b) An arrow $\beta$ is gentle if there is no arrow $\alpha$ such that $\alpha \beta \in I$.

(c) A path $\alpha_{1} \ldots \alpha_{n}$ is formed by consecutive relations if $\alpha_{i} \alpha_{i+1} \in I$ for $1 \leq i<n$.

(d) A path $\alpha_{1} \ldots \alpha_{n}$ is critical if it is formed by consecutive relations and $\alpha_{1}$ is a gentle arrow.

When there is no gentle arrow, we set $n(\Lambda)=0$. When there is a gentle arrow, let $n(\Lambda)$ be the maximal length computed over all critical paths. This number is bounded since $Q$ is finite.

Theorem 2.7 [17] Let $\Lambda=k Q / I$ be a gentle algebra with $n(\Lambda)$ the maximum length over all critical paths. Then inj.dim $\Lambda=n(\Lambda)=\operatorname{proj} \cdot \operatorname{dim} D\left(\Lambda^{o p}\right)$ if $n(\Lambda)>0$, and inj.dim $\Lambda=$ proj.dim $D\left(\Lambda^{o p}\right) \leq 1$ if $n(\Lambda)=0$. In particular, $\Lambda$ is Iwanaga-Gorenstein.

Let $x_{1} \stackrel{\alpha_{1}}{\rightarrow} \cdots \stackrel{\alpha_{n-1}}{\longrightarrow} x_{n} \stackrel{\alpha_{n}}{\longrightarrow} x_{1}$ be a saturated cycle. Let $u_{i}$ be the maximal path avoiding zero-relations starting at the vertex $x_{i}$ and let $v_{i}$ be the the maximal path avoiding zero-relations ending at $x_{i}$. Then the indecomposable projective module $P\left(x_{i}\right)$ and the 
Fig. 1 Local situation for a saturated cycle

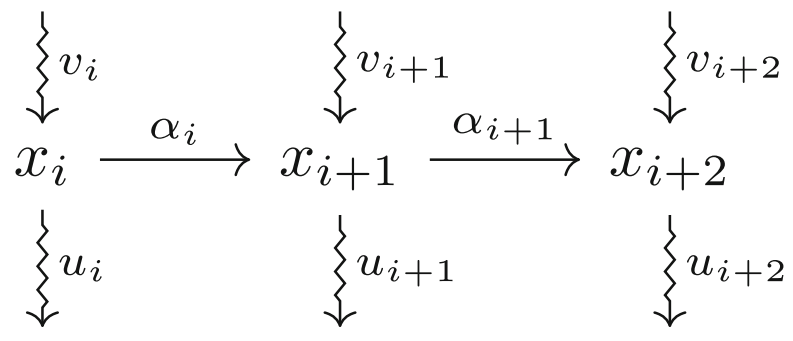

indecomposable injective module $I\left(x_{i}\right)$ are string modules given by $P\left(x_{i}\right)=M\left(u_{i}^{-1} \alpha_{i} u_{i+1}\right)$ and $I\left(x_{i}\right)=M\left(v_{i-1} \alpha_{i-1} v_{i}^{-1}\right)$, see Fig. 1 .

Theorem 2.8 [20, Theorem 2.5] Let $\Lambda=k Q / I$ be a gentle algebra. Let $x_{1} \stackrel{\alpha_{1}}{\longrightarrow} \cdots \stackrel{\alpha_{n-1}}{\longrightarrow}$ $x_{n} \stackrel{\alpha_{n}}{\longrightarrow} x_{1}$ be a saturated cycle. The string module $M\left(u_{i}\right)$, where $u_{i}$ is the string starting at $x_{i}$, is Gorenstein-projective. Moreover, all indecomposable modules in $\underline{\mathrm{GP}}(\Lambda)$ are obtained in such manner.

\section{Monomial 2-Calabi-Yau Tilted Algebras Are Jacobian}

A triangulated $k$-category $\mathcal{C}$, Hom-finite with split idempotents, is $d$-Calabi-Yau if there is a bifunctorial isomorphism $\operatorname{Hom}_{\mathcal{C}}(X, Y) \simeq \operatorname{DHom}_{\mathcal{C}}(Y, X[d])$ for all $X, Y \in \mathcal{C}$. This means that the $d$-th power of [1] is a Serre functor for the category, and this is to say that there is a funtorial isomorphism $\tau^{-1}[d-1]=\mathrm{Id}$.

Definition 3.1 Let $\mathcal{C}$ be a 2-Calabi-Yau category

(1) An object $T$ is cluster-tilting if it is basic and $\operatorname{add} T=\left\{X \in \mathcal{C}: \operatorname{Hom}_{\mathcal{C}}(X, T[1])=0\right\}$.

(2) The endomorphism algebra $\operatorname{End}_{\mathcal{C}}(T)$ of a cluster-tilting object is called a 2-CalabiYau tilted algebra.

Examples of 2-Calabi-Yau tilted algebras are the cluster-tilted algebras defined in [10]. A more general family is obtained from generalized cluster categories defined by Amiot, see [1]. Quivers with potential were introduced in [14]. A potential $W$ is a (possibly infinite) linear combination of cycles in $Q$, up to cyclic equivalence. Given an arrow $\alpha$ and a cycle $c=\alpha_{1} \ldots \alpha_{l}$, the cyclic derivative $\partial_{\alpha}(c)$ is defined by

$$
\partial_{\alpha}\left(\alpha_{1} \ldots \alpha_{l}\right)=\sum_{k+1}^{l} \delta_{\alpha \alpha_{k}} \alpha_{k+1} \ldots \alpha_{l} \alpha_{1} \ldots \alpha_{k-1}
$$

where $\delta_{\alpha \alpha_{k}}$ is the Kronecker delta. This derivative can be applied to a sum of cycles extending by linearity. Notice that a cycle $\alpha_{1} \ldots \alpha_{l}$ may have arrow repetitions. Let $R\langle\langle Q\rangle\rangle$ be the complete path algebra consisting of all (possibly infinite) linear combinations of paths in $Q$. Let $(Q, W)$ be a quiver with potential, the Jacobian algebra is defined to be $\operatorname{Jac}(Q, W)=$ $R\langle\langle Q\rangle\rangle / \overline{\left\langle\partial_{\alpha} W, \alpha \in Q_{1}\right\rangle}$. When one considers the algebra $R\langle\langle Q\rangle\rangle /\left\langle\partial_{\alpha} W, \alpha \in Q_{1}\right\rangle$ and the result is finite dimensional then it is a Jacobian algebra $\operatorname{Jac}(Q, W)$ and there is no need to consider completions and ideal closures. 
Amiot [1, Sec. 3] showed that Jacobian algebras are 2-CY tilted whenever they are finite dimensional by defining a generalized cluster category $\mathcal{C}_{(Q, W)}$. In [2, Question 2.20], the author asked whether all 2-Calabi-Yau tilted algebras are Jacobian, and warned that the answer might be negative in a general setting. The first part of this note is devoted to this question, provided the algebra is monomial.

Now we recall some main properties of 2-Calabi-Yau tilted algebras. The first part of the next theorem is a celebrated result by Keller-Reiten, the second part is a characterization of Gorenstein projective modules that can be computed over the module category.

Theorem 3.2 [16, 21] Let $\Lambda$ be a 2-Calabi-Yau tilted algebra,

(1) $\Lambda$ is 1-Iwanaga-Gorenstein and stably 3-Calabi-Yau.

(2) A $\Lambda$-module $M$ is Gorenstein projective if and only if $\Omega^{2} \tau M \simeq M$ in $\underline{\bmod } \Lambda$.

Let $\Lambda=k Q / I$ be a monomial 2-Calabi-Yau tilted algebra. Since it is a monomial 1-Iwanaga-Gorenstein algebra by the result above, the singularity category $\operatorname{GP}(\Lambda)$ is described in Lemma 2.4. Using this, we can gather information on cycles and relations in $(Q, I)$. Remember that the zero-relations of length $r_{i}$ in $F$ lie in a cycle $c_{i}$ of length $n_{i}$.

Lemma 3.3 Let $\Lambda=k Q /$ I be a monomial 1-Iwanaga-Gorenstein algebra and stably 3Calabi-Yau, and $F=\coprod F_{i}$ the corresponding set of zero-relations. Then $r_{i}=b_{i} n_{i}-1$, where $b_{i} \in \mathbb{Z}^{+}$is such that $r_{i}>0$.

Proof We want to know for which parameters $x, n \in \mathbb{Z}^{+}$we have that an orbit category $\mathcal{D}^{b}\left(\mathbb{A}_{x}\right) / \tau^{n}$ is 3-Calabi-Yau. Recall that in the orbit category [1] and $\tau$ are induced from $\mathcal{D}^{b}\left(\mathbb{A}_{x}\right)$.

So, at least, we need the functor $\tau^{-1}$ [2] to send objects of a certain orbit to objects in the same orbit. One can easily compute that $\tau^{-1}[2] X=\tau^{-x-2} X$ over $\mathcal{D}^{b}\left(\mathbb{A}_{x}\right)$. Hence $\tau^{x+2} X$ has to be $\left(\tau^{n}\right)^{b} X=\operatorname{Id} X=X$ in the orbit category, so $x=n b-2$. If we go back to the notation in Lemma 2.4, the claim follows.

With the last lemma we have restricted the possible lengths of cycles and zero-relations in $k Q / I$, so now there is a constraint for the elements in $F$. In the following we show that a finite dimensional algebra described by a bound quiver $(Q, I)$ satisfying this constraint has to be 2-Calabi-Yau tilted.

Lemma 3.4 Let $\Lambda=k Q / I$ be a 1-Iwanaga-Gorenstein monomial algebra where $F=$ $\bigsqcup_{i \in[1, m]} F_{i}$ is such that $r_{i}=b_{i} n_{i}-1, b_{i} \in \mathbb{Z}^{+}$and $r_{i}>0$. Then the zero-relations arise from a potential $W=\sum_{i \in[1, m]} c_{i}^{b_{i}}$.

Proof Since two different cycles in $\mathcal{C}(\Lambda)$ do not share arrows, each $\alpha \in Q_{1}$ is either in one of the cycles or is not present in $\mathcal{C}(\Lambda)$. If $\alpha$ is not in any cycle then the associated partial derivation adds zero to the ideal $I$. If $\alpha$ is in the cycle $c_{j} \in \mathcal{C}(\Lambda)$ of length $n_{j}$ (with no repetition), then $\partial_{\alpha}\left(\sum_{i} c_{i}^{b_{i}}\right)=\partial_{\alpha}\left(c_{j}^{b_{j}}\right)$ and this is an element of $k Q$ given by $b_{j} u$ where $u$ is a subpath of length $r_{j}=n_{j} b_{j}-1$ in the cycle $c_{j}^{b_{j}}$ without the arrow $\alpha$. Our general hypothesis is that $k$ is a field of characteristic zero, so these paths $b_{j} u$ can be replaced by 
$u$ as generators of $I$. Doing this for all $\alpha \in Q_{1}$ we cover exactly all the elements in $F$. Therefore $\Lambda$ is a Jacobian algebra hence it is 2-Calabi-Yau tilted.

The two lemmas above sum up in the following theorem.

Theorem 3.5 Let $k$ be an algebraically closed field of characteristic zero and $\Lambda=k Q / I$ a monomial $k$-algebra. The following are equivalent:

(1) $\Lambda$ is 2-Calabi-Yau tilted,

(2) $\Lambda$ is a 1-Iwanaga-Gorenstein algebra and stably 3-Calabi-Yau,

(3) $\Lambda$ is Jacobian.

Proof ( $1 \Rightarrow 2$ ) Let $\Lambda$ be monomial 2-Calabi-Yau tilted. By Theorem 3.2 (1) $\Lambda$ is a 1 Iwanaga-Gorenstein algebra with stably 3-Calabi-Yau singularity category.

$(2 \Rightarrow 3$ ) By Lemmas 3.3 and 3.4, a 1-Iwanaga-Gorenstein monomial algebra $\Lambda=k Q / I$ with a 3-Calabi-Yau singularity category is such that the ideal $I$ arises from the cyclic derivatives of a potential, and this potential can be easily defined from $I$.

$(3 \Rightarrow 1)$ It follows from [1, Theorem 3.6] that $\Lambda$ is 2-Calabi-Yau tilted in the sense of Keller-Reiten.

The previous result is written with the restriction: $b_{j} \in \mathbb{Z}^{+}$is non-zero over $k$. To guarantee this we say $k$ is of characteristic zero.

To solve the problem presented by integration-differentiation of potentials over fields of positive characteristic, an alternative is proposed: using hyperpotentials. A hyperpotential on a quiver $Q$ is a collection of elements $\left(\rho_{\alpha}\right)_{\alpha \in Q_{1}}$ over the complete algebra $R\langle\langle Q\rangle\rangle$ such that: for $\alpha: i \rightarrow j, \rho_{\alpha}$ is a (possibly infinite) linear combination of paths $j \rightsquigarrow i$, and $\sum_{\alpha \in Q_{1}}\left[\alpha, \rho_{\alpha}\right]=0$. The Jacobian algebra of a hyperpotential is the quotient $R\langle\langle Q\rangle\rangle / \overline{\left\langle\rho_{\alpha}\right\rangle}$ (see [23, Proposition 1]). In view of this, if we admit hyperpotentials, the last result extends to algebraically closed fields of positive characteristic.

Remark 3.6 Let $\Lambda=k Q / I$ a monomial $k$-algebra, where $k$ is a field of arbitrary characteristic. The following are equivalent:

(1) $\Lambda$ is 2-Calabi-Yau tilted,

(2) $\Lambda$ is a 1-Iwanaga-Gorenstein algebra and stably 3-Calabi-Yau,

(3) $\Lambda$ is a Jacobian algebra of a hyperpotential.

Example 3.7 The algebra $k Q / I$ in Example 2.3 is Jacobian. The potential is $W=\alpha \beta \gamma \delta+$ $\lambda^{5}$.

\subsection{Gentle case}

A particular case of monomial algebras is gentle algebras, see Definition 2.6. In particular gentle Jacobian algebras were studied in [3, Section 2]. There it is proved that a Jacobian algebra arising from a triangulation is gentle and 1-Iwanaga-Gorenstein [3, Theorem 2.7], and a gentle algebra $k Q / I$, where $Q$ has no loops, such that relations are restricted to saturated 3-cycles arises from a surface triangulation.

Now we apply the results in Section 3 but adding the gentle hypothesis. 


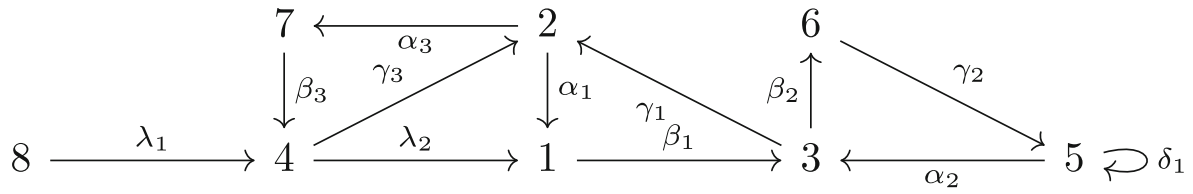

Fig. 2 Gentle bound quiver $(Q, I)$, Example 3.9

Proposition 3.8 Let $k Q /$ I be a gentle 2-Calabi-Yau tilted algebra, then every relation lies on a saturated 3-cycle or a saturated loop.

Proof A gentle algebra is monomial, and by Theorem 3.2 (1) we can apply Lemma 3.3. Then we have $r_{i}=b_{i} n_{i}-1=2$, so $b_{i} n_{i}=3$. We have only two options: $b_{i}=1$ and $n_{i}=3$, or $b_{i}=3$ and $n_{i}=1$. In the first case the relations arise from a potential term of length three $\alpha \beta \gamma$ and we obtain a saturated 3-cycle, and in the second case the potential term is $\delta^{3}$ so we get a loop such that $\delta^{2} \in I$.

We can easily build gentle 2-Calabi-Yau tilted algebras. We only need to be careful so we do not create a cycle that is not in $I$.

Example 3.9 Let $Q$ be the quiver in Fig. 2, and consider the potential $W=\delta_{1}^{3}+$ $\sum_{i=1}^{3} \alpha_{i} \beta_{i} \gamma_{i}$. Then $\operatorname{Jac}(Q, W)$ is a gentle 2-Calabi-Yau tilted algebra.

\section{Other Monomial Algebras and their Singularity Categories}

In this section we study GP for a family of gentle algebras and some examples of nongentle $m$-cluster tilted algebras. We will obtain an analogous property to Theorem 3.2 (2) by explicit computation. This property is deeply related to the CY dimension of GP. ${ }^{1}$

An algebra arising from an $(m+2)$-angulation $\mathcal{T}$ is defined as $k Q_{\mathcal{T}} / I_{\mathcal{T}}$ where the bound quiver $\left(Q_{\mathcal{T}}, I_{\mathcal{T}}\right)$ is obtained from the $(m+2)$-angultion. The set of vertices $\left(Q_{\mathcal{T}}\right)_{0}$ is indexed by the internal $\operatorname{arcs}$ in $\mathcal{T}$. For any two vertices $i, j$, there is an arrow $i \rightarrow j$ when the corresponding $m$-diagonals $i$ and $j$ share a vertex, they are edges of the same $(m+2)$ gon and $i$ follows $j$ clockwise. Given consecutive arrows $i \stackrel{\alpha}{\rightarrow} j \stackrel{\beta}{\rightarrow} k$, then $\alpha \beta \in I_{\mathcal{T}}$ if and only if $i, j$ and $k$ are edges in the same $(m+2)$-gon.

Example 4.1 Draw an annulus as a strip $\Sigma$ and remove a disk from its interior. Add two marked points in this boundary component and other marked points in the boundary of the strip, see Fig. 3. Given a 4-angulation of this marked surface, we can obtain a bound quiver $\left(Q_{\mathcal{T}}, I_{\mathcal{T}}\right)$

In particular, when the surface is a disk (resp. an annulus) the gentle algebra obtained is $m$-cluster tilted of type $\mathbb{A}$ (resp. $\tilde{\mathbb{A}}$ ) in the sense of Thomas [28], as it was studied in several works $[3,5,12,18,26,29]$.

\footnotetext{
${ }^{1}$ This discussion is the main topic of the Appendix. The author is deeply grateful with the Appendix authors for their answer on this subject.
} 

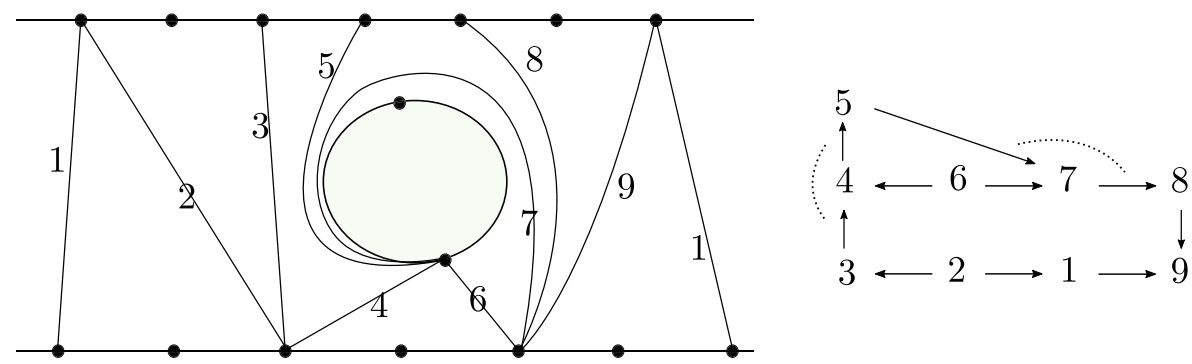

Fig. 3 4-angulation of the sphere with 3 three disks removed, and bound quiver $\left(Q_{\mathcal{T}}, I_{\mathcal{T}}\right)$

The following properties were observed in [26, Rem. 2.18] and [18, Sec. 7] in the case of the disc and annulus, but it is easy to see that they hold when the algebra arises from an $(m+2)$-angulation of a general surface.

Proposition 4.2 Let $\left(Q_{\mathcal{T}}, I_{\mathcal{T}}\right)$ be a bound quiver arising from an $(m+2)$-angulation.

(1) $\Lambda_{\mathcal{T}}=k Q_{\mathcal{T}} / I_{\mathcal{T}}$ is a gentle algebra.

(2) The only possible saturated cycles in $\left(Q_{\mathcal{T}}, I_{\mathcal{T}}\right)$ are $(m+2)$-cycles.

(3) There can be at most $m-1$ consecutive zero-relations not lying in a saturated cycle.

Immediately, we have the following observation.

Lemma 4.3 Let $\Lambda_{\mathcal{T}}=k Q_{\mathcal{T}} / I_{\mathcal{T}}$ be an algebra arising from a $(m+2)$-angulation. Then, $\Lambda_{\mathcal{T}}$ is m-Iwanaga-Gorenstein.

Proof The case $m=1$ follows from Theorem 3.2 (1), and also from [3, Lemma 2.6]. Let $m \geq 2$. Since $\Lambda_{\mathcal{T}}$ is gentle, we can apply Theorem 2.7. First assume that there is no gentle arrow in $\left(Q_{\mathcal{T}}, I_{\mathcal{T}}\right)$, then $n\left(\Lambda_{\mathcal{T}}\right)=0$, so $d$ is zero or one and $d \leq m$. The statement follows.

Now, assume there are gentle arrows in $\left(Q_{\mathcal{T}}, I_{\mathcal{T}}\right)$, and let $\alpha_{1}$ be one of them. It follows that $\alpha_{1}$ is not part of a saturated cycle. Let $\alpha_{1} \ldots \alpha_{r}$ be a critical path. Since $\alpha_{1}$ is not part of a saturated cycle, then none of the arrows $\alpha_{i}$ for $1 \leq i \leq r$ is part of a saturated cycle. By Proposition 4.2 (3), the maximal number of consecutive zero-relations outside of a saturated cycle is $m-1$. Therefore, $r \leq m$, and by Theorem 2.7, $\Lambda_{\mathcal{T}}$ is Gorenstein of dimension $d \leq m$.

Most of the arguments in the following lemma can be found also in [20, Section 4].

Lemma 4.4 Let $\Lambda=k Q /$ I be a gentle d-Iwanaga-Gorenstein algebra, $d \geq 1$. Let $x \in Q_{0}$, and let $N$ be an indecomposable direct summand of $\operatorname{rad} P(x)$. Then,

(a) $\quad N \in \underline{\mathrm{GP}}(\Lambda)$, or

(b) proj.dim $N \leq d-1$. 
Proof If $N$ is projective, we are in case (b). Let $N$ be non projective. Let $P(x)=$ $M\left(u^{-1} \alpha^{-1} \beta w\right)$ be the indecomposable projective and $N=M(u)$ so that $S(t(\alpha))=$ top $M(u)$. We study the cases:

(i) $\quad \alpha$ is part of a saturated cycle $x_{1} \rightarrow \cdots \rightarrow x_{i} \stackrel{\alpha}{\rightarrow} x_{i+1} \cdots \rightarrow x_{1}$.

(ii) $\alpha$ is not part of a saturated cycle.

(i) Let $x_{i} \stackrel{\alpha}{\rightarrow} x_{i+1}$, then $M(u)$ is a direct summand of $\operatorname{rad} P\left(x_{i}\right)$. By Theorem 2.8, $M(u) \in$ $\underline{\mathrm{GP}}(\Lambda)$.

(ii) Since $N=M(u)$ is not projective, there exists an arrow $\delta_{1}$ such that $\alpha \delta_{1} \in I$. The arrow $\delta_{1}$ is not part of a saturated cycle, since then $\alpha$ would be part of the saturated cycle. Let $P(t(\alpha))=M\left(c^{-1} \delta_{1}^{-1} u\right)$, then there is an exact sequence

$$
0 \rightarrow M(c) \rightarrow P(t(\alpha)) \rightarrow M(u) \rightarrow 0 .
$$

If the string module $M(c)$ is not projective, then it satisfies the same conditions as $M(u)$, so we can construct a new exact sequence

$$
0 \rightarrow M\left(c_{1}\right) \rightarrow P\left(t\left(\delta_{1}\right)\right) \rightarrow M(c) \rightarrow 0 .
$$

Recursively, we obtain a path $\alpha \delta_{1} \cdots \delta_{n}$ such that each quadratic factor belongs to $I$. This process has to finish after a finite number of steps, being the direct summand $M\left(c_{n}\right)$ of $P\left(t\left(\delta_{n-1}\right)\right)$ a projective module. If there were not finitely many steps and $M\left(c_{n}\right)$ was not projective, we would find new arrows $\delta_{n+1}, \ldots$ and form a path $\alpha \delta_{1} \cdots \delta_{n} \cdots$ such that each quadratic factor is in $I$. The quiver $Q$ is finite, so the only way to construct an infinite path $\alpha \delta_{1} \cdots \delta_{n} \cdots$ is by reaching a saturated cycle. By the gentleness, if one of the arrows $\delta_{i}$ is in a saturated cycle, then all $\alpha, \delta_{1}, \ldots, \delta_{n}$ are in the saturated cycle, contradicting the condition imposed on $\alpha$. Therefore the procedure to find the short exact sequences stops. Splicing the short exact sequences we get a projective resolution for $M(u)$, which is finite, so proj.dim $M(u)<\infty$. By Remark 2.2, we have proj.dim $M(u) \leq d$. Now, we can also express $M(u)$ as $M(u)=\Omega M(\beta w)$. If we had proj.dim $M(u)=d$, then we would have proj.dim $M(\beta w)=d+1$ and this is impossible by Remark 2.2. Thus, $\operatorname{proj} \cdot \operatorname{dim} M(u) \leq$ $d-1$.

To complete the previous lemma, observe that if $\Lambda$ is selfinjective (that is, $\Lambda$ is 0 -Iwanaga-Gorenstein) then every indecomposable module is Gorenstein-projective.

Theorem 4.5 Let $\Lambda_{\mathcal{T}}=k Q_{\mathcal{T}} I_{\mathcal{T}}$ be an algebra arising from a $(m+2)$-angulation and let $N$ be a $\Lambda_{\mathcal{T}}$-module. Then $N$ is Gorenstein projective if and only if $\Omega^{m+1} \tau N \simeq N$ in $\underline{\bmod } \Lambda_{\mathcal{T}}$. In particular, this holds for $m$-cluster tilted algebras of type $\mathbb{A}$ and $\tilde{\mathbb{A}}$.

Proof Let $M$ be an indecomposable module in $\underline{\mathrm{GP}}\left(\Lambda_{\mathcal{T}}\right)$. By Theorem 2.8, $M=M\left(u_{i}\right)$ where $u_{i}$ is the maximal non-zero path starting at $x_{i}$.

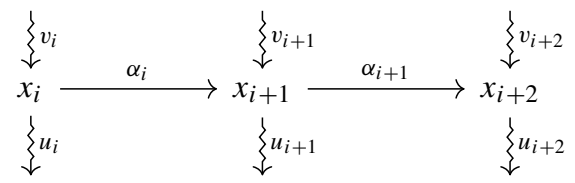


We compute a minimal projective presentation of $M\left(u_{i}\right)$.

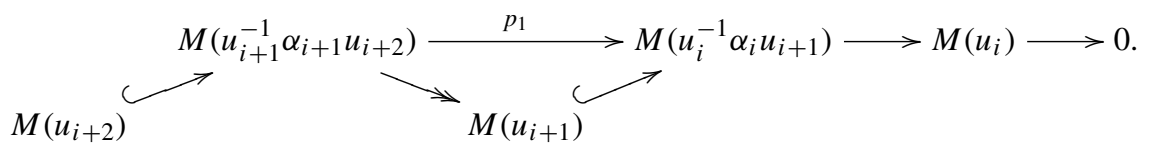

Observe that $\Omega^{t} M\left(u_{i}\right)=M\left(u_{i+t}\right)$, where $t$ is an integer considered modulo $m+2$. Applying the Nakayama functor we get

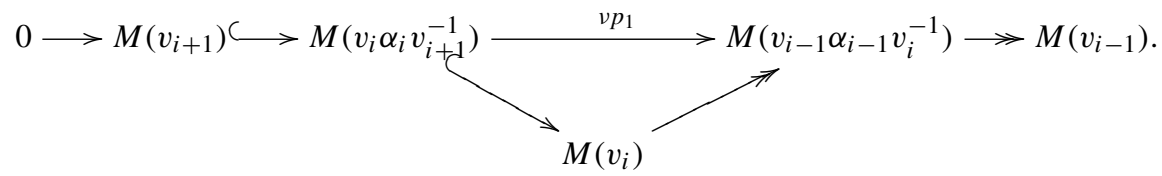

Then $\tau M\left(u_{i}\right)=\operatorname{ker} v p_{1}=M\left(v_{i+1}\right)$. Let $S\left(y_{i+1}\right)=\operatorname{top} M\left(v_{i+1}\right)$. Let $P\left(y_{i+1}\right)=$ $M\left(w_{i+1}^{-1} v_{i+1} \alpha_{i+1} u_{i+2}\right)$ be the projective cover of $M\left(v_{i+1}\right)$.

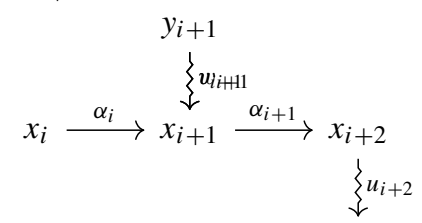

Therefore, $\Omega M\left(v_{i+1}\right)=M\left(w_{i+1}^{\prime}\right) \oplus M\left(u_{i+2}\right)$, where $M\left(w_{i+1}^{\prime}\right)$ is the maximal submodule of $M\left(w_{i+1}\right)$. The syzygy functor is additive, so

$$
\Omega^{m+1} \tau M\left(u_{i}\right)=\Omega^{m+1} M\left(v_{i+1}\right)=\Omega^{m} M\left(w_{i+1}^{\prime}\right) \oplus \Omega^{m} M\left(u_{i+2}\right) .
$$

Since $\Omega^{t} M\left(u_{i}\right)=M\left(u_{i+t}\right)$, we have

$$
\Omega^{m} M\left(w_{i+1}^{\prime}\right) \oplus \Omega^{m} M\left(u_{i+2}\right)=\Omega^{m} M\left(w_{i+1}^{\prime}\right) \oplus M\left(u_{i}\right) .
$$

Now, we only need to prove that $\Omega^{m} M\left(w_{i+1}^{\prime}\right)=0$. Observe that $M\left(w_{i+1}^{\prime}\right)$ is a direct summand of $\operatorname{rad} P\left(y_{i+1}\right)$.

We know by Lemma 4.3 that $\Lambda_{\mathcal{T}}$ is Gorenstein of dimension $d \leq m$. By Lemma 4.4 one of the following holds:

(1) $\operatorname{proj} \cdot \operatorname{dim} M\left(w_{i+1}^{\prime}\right) \leq m-1$, or

(2) $M\left(w_{i+1}^{\prime}\right) \in \underline{\mathrm{GP}}\left(\Lambda_{\mathcal{T}}\right)$.

If (1) holds, then $\Omega^{m} M\left(w_{i+1}^{\prime}\right)=0$ and we are done.

We assume (2) holds, so $M\left(w_{i+1}^{\prime}\right) \in \underline{\mathrm{GP}}\left(\Lambda_{\mathcal{T}}\right)$. We prove that this leads to a contradiction. Let $z_{i+1}$ be the vertex such that top $M\left(w_{i+1}^{\prime}\right)=S\left(z_{i+1}\right)$. By the description in Theorem 2.8, the vertex $z_{i+1}$ is the target of an arrow $\gamma$ in a saturated $(m+2)$-cycle and $\gamma w_{i+1}^{\prime} \neq 0$.

(2a) If the arrow $\gamma$ is $y_{i+1} \stackrel{\gamma}{\rightarrow} z_{i+1}$, as appears in the figure below, then there is an arrow $a_{j}$ in the saturated $(m+2)$-cycle, such that $a_{j} \gamma \in I_{\mathcal{T}}$. Then, $a_{j} v_{i+1} \neq 0$, 
contradicting that $I\left(x_{i+1}\right)=M\left(v_{i} \alpha_{i} v_{i+1}^{-1}\right)$ is the indecomposable injective associated to $x_{i}$. This is absurd.

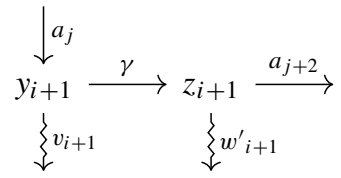

(2b) If the arrow $\gamma$ in a saturated cycle is such that $s(\gamma) \neq y_{i+1}$, as we see in the figure below, there is an arrow $b_{j+2}$ following the saturated cycle such that $\gamma b_{j+2} \in I_{\mathcal{T}}$. Thus, we have $\gamma w_{i+1}^{\prime} \neq 0$ and by gentleness, $a_{j+1} b_{j+2} \notin I_{\mathcal{T}}$.

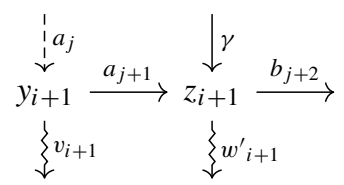

But recall that $M\left(w_{i+1}^{\prime}\right)$ is a submodule of $\operatorname{rad} P\left(y_{i+1}\right)$, so $b_{j+2}$ has to be the first arrow in the string $w_{i+1}^{\prime}$. This contradicts that $M\left(w_{i+1}^{\prime}\right)$ is a submodule of $\operatorname{rad} P\left(y_{i+1}\right)$. To sum up, $M\left(w_{i+1}^{\prime}\right)$ is not a trivial Gorenstein projective module and just (1) holds.

As a corollary, we obtain the next result that generalizes the properties known for clustertilted algebras: Theorem 3.2 (1) and (2).

Corollary 4.6 Let $\Lambda$ be a m-cluster tilted algebra of type $\mathbb{A}$ or $\tilde{\mathbb{A}}$. Then

(1) $\Lambda$ is $m$-Iwanaga-Gorenstein, and

(2) $N \in \operatorname{GP}(\Lambda)$ if and only if $\Omega^{m+1} \tau N \cong N$.

Proof Part (1) follows from Lemma 4.3. Part (2) follows from Theorem 4.5.

From a more general point of view, $m$-cluster tilted algebras are $(m+1)$-Calabi-Yau tilted algebras. It is known, and expected, that Corollary 4.6 does not hold in general for $d$-Calabi-Yau tilted algebras by the next reasons:

(1) In [21, Section 5.3] there is an example (due to Iyama) of a $d$-Calabi-Yau tilted algebra that is not Iwanaga-Gorenstein.

(2) Moreover, a recent preprint [24] shows that all finite dimensional $k$-algebras are $d$ Calabi-Yau tilted (for all $d>2$ ).

Still, there are results in this direction due to Keller and Reiten [22, Section 4.6], and Beligiannis [7, Theorem 6.4], showing that a $d$-Calabi-Yau tilted algebra is Iwanaga-Gorenstein under certain conditions. In both cases, it is required that add $T$ is corigid to some degree $u$, meaning that $\operatorname{Hom}_{\mathcal{C}}(T, T[-t])=0$ for all $1 \leq t \leq u$. Over $m$-cluster tilting categories of type $\mathbb{A}$ or $\tilde{\mathbb{A}}$, the full subcategory defined by a cluster tilting object add $T$ is $(m+1)$ cluster tilting. In the next example we show that Corollary 4.6 is independent of these 


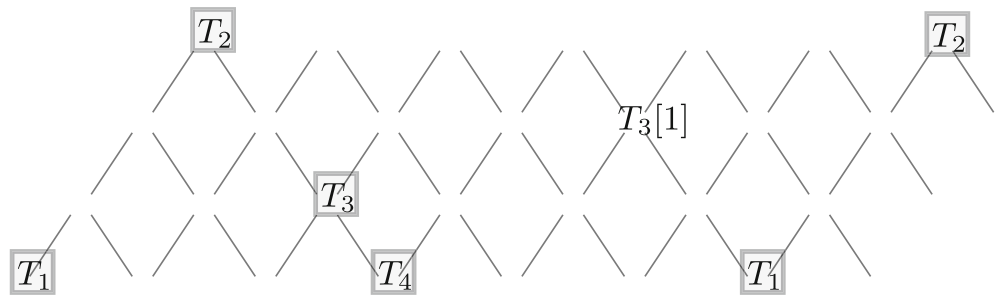

Fig. 4 A 2-cluster tilting object $T$ which is not corigid

results, by giving an example of a non-corigid cluster-tilting object in the 2-cluster category of type $\mathbb{A}_{4}$.

Example 4.7 Let $\mathcal{C}_{Q}^{2}$ be the 2-cluster category, where $Q$ is of type $\mathbb{A}_{4}$ and let $T$ be as in Fig. 4. The object $T$ is not corigid since $\operatorname{Hom}\left(T_{3}, T_{1}[-1]\right) \simeq \operatorname{Hom}\left(T_{3}[1], T_{1}\right) \neq 0$. By Corollary 4.6 the 2-cluster tilted algebra $\operatorname{End}(T)$ is 2-Iwanaga-Gorenstein. In fact, in this example the algebra, given by the quiver below bound by $\beta \alpha=0$, is of global dimension two.

$$
1 \longleftarrow \alpha \longleftarrow \beta
$$

In the next example we see that there are $m$-cluster-tilted algebras not arising from $(m+$ 2)-angulations, defined by non-corigid cluster tilting objects, for which the conclusion of Corollary 4.6 holds.

Example 4.8 Let $\mathcal{C}_{Q}^{2}$ be the 2-cluster category of type $\mathbb{D}_{6}$, and $T=\bigoplus_{i=1}^{6} T_{i}$ the 2-cluster tilting object in Fig. 5. The algebra. $\Lambda=\operatorname{End}_{\mathcal{C}_{Q}^{2}}(T)$ in Example 4.8 is given by the quiver in Fig. 6 and the ideal $I=\langle\lambda \alpha, \alpha \beta \gamma, \beta \gamma \delta, \delta \lambda\rangle$. We see that $\Lambda$ is 2-Iwanaga-Gorenstein and has infinite global dimension. The indecomposable modules in $\underline{\mathrm{GP}}(\Lambda)$ are $3,6,5,2,2$, exactly those such that $\Omega^{3} \tau N=N$.

As a final remark, we may ask the next question:

Question 4.9 $m$-cluster tilted algebras are still a special subclass of $(m+1)$-Calabi-Yau tilted algebras. Are they always Iwanaga-Gorenstein? Does Corollary 4.6 always hold for these algebras?

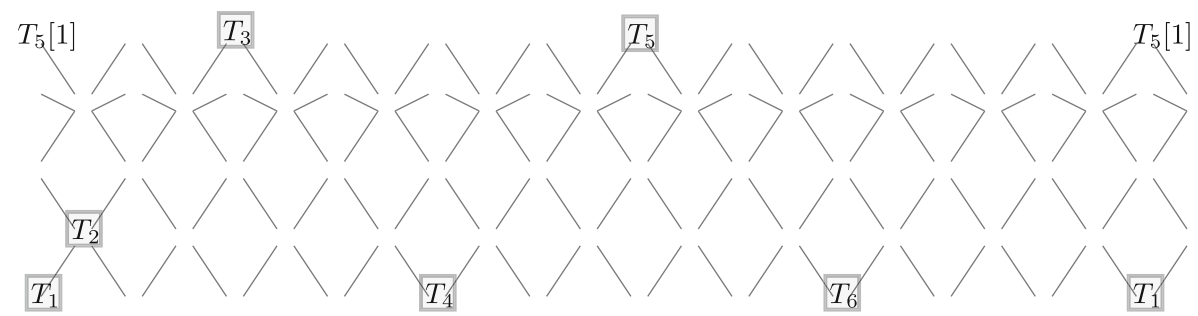

Fig. 5 A 2-cluster tilting object $T$ such that $\operatorname{Hom}\left(T_{5}, T_{3}[-1]\right)=\operatorname{Hom}\left(T_{5}[1], T_{3}\right) \neq 0$, so $T$ is not corigid 
Fig. $6 \Lambda=k Q / I$, Example 4.8
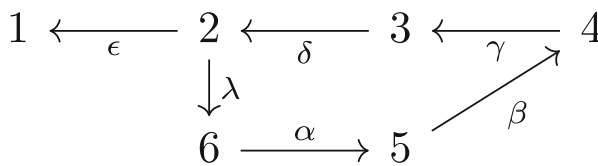

Acknowledgments This work was supported by the Austrian Science Fund Project Number P25647- N26. The author wants to thank the researchers at Universität Stuttgart, especially Prof. Steffen Koenig and Dr. Matthew Pressland, and also would like to thank the organizers of BIREP-2017 Summer School on Gentle Algebras.

Funding Information Open access funding provided by University of Graz.

Open Access This article is licensed under a Creative Commons Attribution 4.0 International License, which permits use, sharing, adaptation, distribution and reproduction in any medium or format, as long as you give appropriate credit to the original author(s) and the source, provide a link to the Creative Commons licence, and indicate if changes were made. The images or other third party material in this article are included in the article's Creative Commons licence, unless indicated otherwise in a credit line to the material. If material is not included in the article's Creative Commons licence and your intended use is not permitted by statutory regulation or exceeds the permitted use, you will need to obtain permission directly from the copyright holder. To view a copy of this licence, visit http://creativecommonshorg/licenses/by/4.0/.

\section{Appendix A: Auslander-Reiten translations for Gorenstein algebras by Sondre Kvamme and Matthew Pressland}

Let $\Lambda$ be an Iwanaga-Gorenstein algebra. Since $\operatorname{GP}(\Lambda)$ is a functorially finite subcategory of $\bmod \Lambda$, it has Auslander-Reiten sequences [30], inducing an Auslander-Reiten translation $\tau_{\mathrm{GP}}$ on $\underline{G P}(\Lambda)$, typically different from the Auslander-Reiten translation $\tau_{\Lambda}$ on $\underline{\bmod } \Lambda$. The goal of this appendix is to relate the objects $\tau_{\Lambda} M$ and $\tau_{\mathrm{GP}} M$ of $\underline{\bmod } \Lambda$ when $M$ is Gorenstein projective. Indeed, we will show that these objects eventually coincide after repeated application of the syzygy functor. While this fact may not be surprising to experts, and can be deduced quickly from results already in the literature (most easily from [31, Thm. 3.7]), we give here a very direct proof, which even exhibits a natural isomorphism of functors. Moreover, we explain how this result provides a new perspective on results of Garcia Elsener (Corollary 4.6 of the present paper) and Garcia Elsener-Schiffler [32, Thm. 1] characterising Gorenstein projective modules over certain Calabi-Yau tilted algebras $\Lambda$, by relating these characterisations directly to a Calabi-Yau property of $\underline{G P}(\Lambda)$.

We denote by $\Omega: \underline{\bmod } \Lambda \rightarrow \underline{\bmod } \Lambda$ the syzygy functor, taking a module to the kernel of a projective cover, and by $\Sigma: \underline{\bmod \Lambda} \rightarrow \underline{\bmod \Lambda}$ its left adjoint, taking a module to the cokernel of a right proj $\Lambda$-approximation. The restrictions of these functors to $\underline{G P}(\Lambda)$ are mutually inverse, the restriction of $\Sigma$ being the suspension functor on this triangulated category.

Lemma A.1 The functor $\Sigma^{d}: \underline{\bmod } \Lambda \rightarrow \underline{\bmod } \Lambda$ has essential image in $\underline{G P}(\Lambda)$.

Proof Since $\Lambda$ is $d$-Iwanaga-Gorenstein, we have $\Omega^{d} \Sigma^{d} M \in G P(\Lambda)$ for any $\Lambda$-module $M$. Then $\Sigma^{d} \Omega^{d} \Sigma^{d} M \in \underline{G P}(\Lambda)$ since $\Sigma$ preserves Gorenstein projectivity. It follows from the triangular identities for the unit and counit of the adjoint pair $\left(\Sigma^{d}, \Omega^{d}\right)$ that $\Sigma^{d} M$ is a summand of $\Sigma^{d} \Omega^{d} \Sigma^{d} M$, and hence is itself Gorenstein projective. 
Theorem A.2 Let $\Lambda$ be a finite-dimensional Iwanaga-Gorenstein algebra of Gorenstein dimension at most $d$. Then there is a natural isomorphism

$$
\Omega^{d} \tau_{\Lambda} \simeq \Omega^{d} \tau_{\mathrm{GP}}
$$

of endofunctors of $\underline{G P}(\Lambda)$.

Proof Using the Yoneda embedding, it suffices to show that there is a natural isomorphism

$$
\underline{\operatorname{Hom}}_{\Lambda}\left(-, \Omega^{d} \tau_{\mathrm{GP}} X\right) \cong \underline{\operatorname{Hom}}_{\Lambda}\left(-, \Omega^{d} \tau_{\Lambda} X\right)
$$

of functors on $\underline{\bmod } \Lambda$, for any $X \in \operatorname{GP}(\Lambda)$. By adjunction and the Auslander-Reiten formula for $\operatorname{GP}(\Lambda)$, we obtain natural isomorphisms

$$
\underline{\operatorname{Hom}}_{\Lambda}\left(-, \Omega^{d} \tau_{\mathrm{GP}} X\right) \cong \underline{\operatorname{Hom}}_{\Lambda}\left(\Sigma^{d}-, \tau_{\mathrm{GP}} X\right) \cong \operatorname{DExt}_{\Lambda}^{1}\left(X, \Sigma^{d}-\right),
$$

The validity of the second isomorphism depends on Lemma A.1, showing that $\Sigma^{d}$ takes values in $\underline{G P}(\Lambda)$. Alternatively, using the Auslander-Reiten formula in $\bmod \Lambda$, we get

$$
\underline{\operatorname{Hom}}_{\Lambda}\left(-, \Omega^{d} \tau_{\Lambda} X\right) \cong \underline{\operatorname{Hom}}_{\Lambda}\left(\Sigma^{d}-, \tau_{\Lambda} X\right) \cong \operatorname{DExt}_{\Lambda}^{1}\left(X, \Sigma^{d}-\right) .
$$

The two right-hand sides coincide, completing the proof.

Theorem A.3 ([32, Thm. 1]) Let $\Lambda$ be a 2-Calabi-Yau tilted algebra. Then a $\Lambda$-module $M$ is Gorenstein projective if and only if $M \cong \Omega^{2} \tau_{\Lambda} M$ in $\underline{\bmod \Lambda}$.

Proof By a result of Keller and Reiten [21, Prop. 2.1], a 2-Calabi-Yau tilted algebra is Iwanaga-Gorenstein of Gorenstein dimension at most 1 . Thus $\Omega^{2} N \in \underline{G P}(\Lambda)$ for any $N \in \bmod \Lambda$, and the 'if' part of the theorem follows immediately.

For the 'only if' direction, Keller and Reiten also prove [21, Thm. 3.3] that $G P(\Lambda)$ is a 3Calabi-Yau category, meaning that $\Sigma^{3}$ is a Serre functor. On the other hand, the AuslanderReiten formula states that $\Sigma \tau_{\mathrm{GP}}$ is always a Serre functor on $\underline{G P}(\Lambda)$. Since Serre functors are unique up to natural isomorphism, the 3-Calabi-Yau property is equivalent to there being a natural isomorphism $\tau_{\mathrm{GP}} \simeq \Sigma^{2}$. Now by Theorem A.2, using again the IwanagaGorenstein property of $\Lambda$, we have

$$
\Omega^{2} \tau_{\Lambda} M \cong \Omega^{2} \tau_{\mathrm{GP}} M \cong \Omega^{2} \Sigma^{2} M \cong M .
$$

We close by observing that characterisations of Gorenstein projective $\Lambda$-modules as in Theorem A.3 are closely related to Calabi-Yau properties of $\underline{G P}(\Lambda)$ in wider generality.

Proposition A.4 Let $\Lambda$ be Iwanaga-Gorenstein of Gorenstein dimension at most $m$. Then $\underline{G P}(\Lambda)$ is $(m+1)$-Calabi-Yau if and only if there is a natural isomorphism $\Omega^{m} \tau_{\Lambda} \simeq$ $\operatorname{Id}_{G P}(\Lambda)$. In this case, a $\Lambda$-module $M$ is Gorenstein projective if and only if $M \cong \Omega^{m} \tau_{\Lambda} M$ in $\underline{\bmod \Lambda}$.

Proof As in the proof of Theorem A.2, $G P(\Lambda)$ is $(m+1)$-Calabi-Yau if and only if there is a natural isomorphism $\tau_{\mathrm{GP}} \simeq \Sigma^{m}$ on $\underline{G P}(\Lambda)$. Since $\Sigma^{m}$ has quasi-inverse $\Omega^{m}$ on this category, this is equivalent to asking that $\bar{\Omega}^{m} \tau_{\mathrm{GP}} \simeq \operatorname{Id}_{G P(\Lambda)}$. But Theorem A.2 tells us that $\Omega^{m} \tau_{\mathrm{GP}} \simeq \Omega^{m} \tau_{\Lambda}$ in this setting, and the first part of the statement follows. The second part is then proved as in Theorem A.3, replacing 2 by $m$. 
Note that the isomorphisms constructed in the proof of Theorem A.3 arise from a natural isomorphism in this way, using the 3-Calabi-Yau property of $G P(\Lambda)$ [21, Thm. 3.3]. We also deduce that a similar Calabi-Yau property holds for certain gentle $m$-cluster-tilted algebras.

Corollary A.5 Let $\Lambda$ be an $m$-cluster-tilted algebra of type $\mathbb{A}$ or $\tilde{\mathbb{A}}$. Then $\underline{G P}(\Lambda)$ is $(m+$ 1)-Calabi-Yau.

Proof By Corollary 4.6, $\Lambda$ is Iwanaga-Gorenstein of Gorenstein dimension at most $m$, and $M \cong \Omega^{m} \tau_{\Lambda} M$ for each $M \in \operatorname{GP}(\Lambda)$. Since $\Lambda$ is a gentle algebra, meaning that $\underline{G P}(\Lambda)$ is semi-simple [33], there must be a collection of such isomorphisms that is natural in $M-$ for example, by first choosing such an isomorphism for each $M$ in a set of representatives for the isoclasses of indecomposable objects, and then extending to arbitrary objects by choosing direct sum decompositions. (We use here that the ground field $k$ is algebraically closed, so that each indecomposable has endomorphism ring $k$.) The result then follows by Proposition A.4.

\section{References}

1. Amiot, C.: Cluster categories for algebras of global dimension 2 and quivers with potential. Ann. Inst. Fourier 59(6), 2525-2590 (2009)

2. Amiot, C.: On generalized cluster categories, Representations of Algebras and Related Topics, EMS Ser. Congr. Rep. Eur. Math. Soc., Zürich, (16-02), pp. 1-53 (2011)

3. Assem, I., Brüstle, T., Charbonneau-Jodoin, G., Plamondon, P.G.: Gentle algebras arising from surface triangulations. Algebr. Number Theory 4(2), 201-229 (2010)

4. Assem, I., Simson, D., Skowroński, A.: Elements of the Representation Theory of Associative Algebras, London Math. Soc. Student Texts, vol. 65. Cambridge University Press (2006)

5. Baur, K., Marsh, R.: A geometric description of $m$-cluster categories. Trans. Amer. Math. Soc. 360(11), 5789-5803 (2008)

6. Beligiannis, A.: The homological theory of contravariantly finite subcategories: Auslander-buchweitz contexts Gorenstein categories and (co-)stabilization. Comm. Algebra 28(10), 4547-4596 (2000)

7. Beligiannis, A.: Relative homology, higher cluster-tilting theory and categorified Auslander-Iyama correspondence. J. Algebra 444, 367-503 (2015)

8. Burban, I., Iyama, O., Keller, B., Reiten, I.: Cluster tilting for one-dimensional hypersurface singularities. Adv. Math. 217(6), 2443-2484 (2008)

9. Buan, A., Marsh, R., Reineke, M., Reiten, I., Todorov, G.: Tilting theory and cluster combinatorics. Adv. Math. 204(2), 572-518 (2006)

10. Buan, A., Marsh, R., Reiten, I.: Cluster-tilted algebras. Trans. Amer. Math. Soc. 359(1), 323-332 (2007)

11. Butler, M.C.R., Ringel, C.M.: Auslander-reiten sequences with few middle terms and applications to string algebras. Commun. Algebra 15(1-2), 145-179 (1987)

12. Caldero, P., Chapoton, F., Schiffler, R.: Quivers with relations arising from clusters $\left(a_{n}\right.$ case). Trans. Amer. Math. Soc. 358(3), 1347-1364 (2006)

13. Chen, X.W., Shen, D., Zhou, G.: The Gorenstein-projective modules over a monomial algebra. Proc. R. Soc. Edinburgh Sect. Math. 148(6), 1115-1134 (2018)

14. Derksen, H., Weyman, J., Zelevinsky, A.: Quivers with potentials and their representations I: Mutations. Sel. Math. 14(1), 59-119 (2008)

15. Fomin, S., Zelevinsky, A.: Cluster algebras i: foundations. J. Am. Math. Soc. 15(2), 497-529 (2002)

16. Garcia Elsener, A., Schiffler, R.: On syzygies over 2-Calabi-Yau tilted algebras. J. Algebra 470, 91-121 (2017)

17. Geiss, Ch., Reiten, I.: Gentle Algebras are Gorenstein. Representations of algebras and related topics (45), 129 (2005)

18. Gubitosi, V.: $M$-cluster tilted algebras of type Ã. Comm. Algebra 46(8), 3563-3590 (2018)

19. Happel, D., Ringel, C.M.: Tilted algebras. Trans. Am. Math. Soc. 274(2), 399-443 (1982) 
20. Kalck, M.: Singularity categories of gentle algebras. Bulle Lond Math Soc 47(1) (2014)

21. Keller, B., Reiten, I.: Cluster-tilted algebras are Gorenstein and stably Calabi-Yau. Adv. Math. 211(1), 123-151 (2007)

22. Keller, B., Reiten, I.: Acyclic Calabi-Yau categories. Compos. Math. 144(5), 1332-1348 (2008)

23. Ladkani, S.: 2-CY-tilted algebras that are not Jacobian. arXiv:1403.6814 (2014)

24. Ladkani, S.: Finite-dimensional algebras are ( $m>2)$-Calabi-Yau-tilted. arXiv:1603.09709 (2016)

25. Lu, M., Zhu, B.: Singularity categories of Gorenstein monomial algebras. arXiv:1708.00311 (2017)

26. Murphy, G.J.: Derived equivalence classification of $m$-cluster tilted algebras of type An. J. Algebra 323(4), 920-965 (2010)

27. Reiten, I.: Calabi-yau tilted algebras. Sao Paulo J. Math. Sci. 4(3), 529-545 (2010)

28. Thomas, H.: Defining an $m$-cluster category. J. Algebra 318(1), 37-46 (2007)

29. Torkildsen, H.A.: A Geometric Realization of the $m$-cluster Category of Affine Type A. Comm. Algebra 43(6), 2541-2567 (2015)

30. Auslander, M., Smalø, S.: Almost split sequences in subcategories. J. Algebra 2, 426-454 (1981)

31. Auslander, M., Reiten, I.: Cohen-macaulay and Gorenstein Artin algebras. Progr. Math. 95, 221-245 (1991)

32. Garcia Elsener, A., Schiffler, R.: On syzygies over 2-Calabi-Yau tilted algebras. J. Algebra 470, 91-121 (2017)

33. Kalck, M.: Singularity categories of gentle algebras. Bull. Lond. Math. Soc. 47(1), 65-74 (2015)

Publisher's Note Springer Nature remains neutral with regard to jurisdictional claims in published maps and institutional affiliations. 